\title{
The synovium and synovial fluid in multicentric reticulohistiocytosis - a light microscopic, electron microscopic and cytochemical analysis of one case
}

\author{
AJ FREEMONT, CJP JONES, ${ }^{*}$ J DENTON* \\ From the Departments of Pathology and *Rheumatology, University of Manchester and Hope Hospital, \\ Manchester
}

SUMMARY The synovium and synovial fluid have been studied in a patient with multicentric reticulohistiocytosis. Previously unreported histochemical, immunocytochemical and ultrahistochemical findings are presented and their relevance to the aetiology and pathogenesis are discussed. The synovial fluid analysis in this disease is characteristic and may be helpful in its early diagnosis.

Multicentric reticulohistiocytosis is a rare disease of unknown aetiology and pathogenesis which presents clinically with a polyarthropathy, mucocutaneous nodular pruritic eruptions and constitutional symptoms. In approximately $25 \%$ of patients it is associated with an underlying malignancy ${ }^{1}$ and in the remainder the arthropathy frequently becomes severely destructive and unresponsive to treatment. Despite the seriousness of the condition, a retrospective study has demonstrated how easily the disease can be misdiagnosed ${ }^{2}$ both clinically and histologically.

Patients with multicentric reticulohistiocytosis commonly present with pain and swelling of the joints-days, months and even years before the onset of other clinical manifestations ${ }^{3}$ and it is at this stage that the diagnosis is most easily overlooked.

In this report findings from the study of the synovial fluid and synovium from a case of multicentric reticulohistiocytosis are detailed and hitherto undescribed features of both are presented which, because they are unique to this condition, may assist in its early diagnosis.

\section{Case history}

A 72-year-old man with a past history of exposure to asbestos, presented with a four month history of painful, stiff proximal and distal interphalangeal, metacarpophalangeal, wrist and knee joints and bilateral knee effusions; at presentation the arthropathy was associated with multiple papulonodular skin lesions, and general malaise. The effusions were aspirated and an arthroscopic synovial biopsy taken from the left knee. On the basis of the clinical and pathological findings, a diagnosis of multicentric reticulohistiocytosis was made.

Because of suspicious appearances on routine chest $x$-ray examination, the history of asbestos exposure and the diagnosis of multicentric reticulohistiocytosis, the patient underwent investigation for an underlying malignant neoplasm. The presence of a mesothelioma was eventualy demonstrated by pleural biopsy.

\section{Material and methods}

SYNOVIAL FLUID

Specimens of synovial fluid were processed using the routine methods employed in this laboratory. After visual examination of the heparinised sample, a few drops of fluid were placed on a slide and examined for rhagocyte inclusions. A $0.5 \mathrm{ml}$ aliquot was then mixed with $5 \mathrm{ml} 2 \%$ acetic acid and stirred to assess mucin clot formation. Fluid was also diluted, stained with methyl violet and total numbers of cells assessed in a Fuchs-Rosenthal counting chamber.

Cell monolayers were prepared by cytocentrifugation, some were fixed in methanol, stained using a standard Jenner-Giesma method and mounted in synthetic resin, others were stained unfixed for non-specific esterase using the method of $\mathrm{Yam}^{4}$ and mounted in Glassbond.

Cells were classified using the following criteria: Rhagocytes: cells which when viewed unfixed and unstained in transmitted light, contain refractile, non-birefringent, apple-green cytoplasmic granules. 
Lymphocytes: any cells $\leqslant 12 \mu \mathrm{m}$ in diameter with a nuclear to cytoplasmic ratio of more than $80 \%$. Lymphoblasts: any cells, not exhibiting the properties of monocytes (vide infra), $>12 \mu \mathrm{m}$ in diameter, with a nuclear to cytoplasmic ratio greater than $50 \%$.

$\mathrm{T}$ lymphocytes and lymphoblasts: lymphocytes and lymphoblasts containing a few non-specific esterase positive cytoplasmic granules, B lymphocytes and lymphoblasts being all those which are negative for non-specific esterase. ${ }^{5}$

Monocytes: any cells displaying the typical strong macrophage staining reaction with non-specific esterase.

\section{SYNOVIAL BIOPSY}

The synovial biopsy was divided into two parts. One portion was fixed in $10 \%$ neutral buffered formalin and embedded in Paraplast wax. Sections $4 \mu \mathrm{m}$ thick were stained with haematoxylin and eosin (HE) and periodic acid-Schiff (PAS), both with and without pretreatment with diastase, using standard techniques. Other $4 \mu \mathrm{m}$ thick sections were trypsinised in a solution containing $0.1 \%$ trypsin (Sigma) and $0.1 \%$ calcium chloride in Tris-buffered saline $\mathrm{pH}$ 7.8 at $37^{\circ} \mathrm{C}$ for $20 \mathrm{~min}$. Muramidase and alpha-1antitrypsin activity were then demonstrated by a standard immunoperoxidase technique using rabbit primary antibodies and a peroxidase-antiperoxidase (PAP) method with diaminobenzidine to localise peroxidase activity as described by Graham and Karnovsky. ${ }^{6}$

Part of the second portion was cut into small pieces and fixed for $4 \mathrm{~h}$ in $2.5 \%$ glutaraldehyde in $0.1 \mathrm{M}$ cacodylate buffer ( $\mathrm{pH} \mathrm{7.3)}$ at room temperature. These pieces of tissue were then diced into $1 \mathrm{~mm}$ cubes, postfixed in $1 \%$ osmium tetroxide in cacodylate buffer at $4^{\circ} \mathrm{C}$ for $1 \mathrm{~h}$, dehydrated in graded alcohols and propylene oxide and embedded in TAAB resin. Sections $0.5 \mu \mathrm{m}$ thick were cut and stained with $1 \%$ toluidine blue in borax and suitable areas selected for ultrathin sectioning. Grids were double stained with uranyl acetate and Reynold's lead citrate and examined in a Philips 301 electron microscope. The remainder of the second portion of synovium was processed for ultrahistochemistry. Both aryl sulphatase ${ }^{7}$ and acid phosphatase ${ }^{8}$ activity were detected by methods already described.

\section{Results}

SYNOVIAL FLUID

The synovial fuid analysis (Table) showed the following notable features:-

1 A low cell count.

2 Poor or absent mucin clot formation.
Synovial fuid analyses

\begin{tabular}{|c|c|c|}
\hline & \multicolumn{2}{|l|}{ Knee } \\
\hline & Right & Left \\
\hline $\begin{array}{l}\text { Appearance } \\
\text { Mucin clot } \\
\text { WBC (cells/cm }{ }^{3} \text { ) } \\
\text { Rhagocytes \% } \\
\text { Neutrophils \% } \\
\text { Lymphocytes }\end{array}$ & $\begin{array}{l}\text { Bloodstained } \\
\text { Nil } \\
220 \\
<1 \\
18\end{array}$ & $\begin{array}{l}\text { Bloodstained } \\
\text { Poor } \\
520 \\
10 \\
9\end{array}$ \\
\hline $\begin{array}{l}\text { Total \% } \\
\text { B \% } \\
\text { T \% }\end{array}$ & $\begin{array}{l}35 \\
30 \\
5\end{array}$ & $\begin{array}{l}42 \\
30 \\
12\end{array}$ \\
\hline $\begin{array}{l}\text { Lymphoblasts } \\
\text { Total \% } \\
\text { B \% } \\
\text { T \% } \\
\text { Monocytes \% } \\
\text { Synoviocytes \% } \\
\text { Others \% } \\
\text { Crystals }\end{array}$ & $\begin{array}{l}9 \\
6 \\
3 \\
37 \\
1 \\
1 \text { megalocyte } \\
\text { Nil }\end{array}$ & $\begin{array}{l}12 \\
7 \\
5 \\
36 \\
0 \\
1 \text { megalocyte } \\
\text { Nil }\end{array}$ \\
\hline
\end{tabular}

3 The relative proportions of lymphocytes, monocytes and neutrophils.

4 The presence of bizarre megalocytes (Fig. 1); these cells were $50-70 \mu \mathrm{m}$ in diameter, each containing a large 20-30 $\mu \mathrm{m}$ nucleus and abundant cytoplasm which showed the diffuse granular nonspecific esterase staining pattern characteristic of cells of the monocyte/macrophage lineage.

\section{SYNOVIUM}

\section{Light microscopy}

The synovial intima showed patchy areas of synoviocyte hyperplasia beneath which were many layers of infiltrating large cells, $50-100 \mu \mathrm{m}$ in diameter, with abundant eosinophilic finely granular cytoplasm (Fig. 2). Although the majority of these megalocytes were mononuclear, some were multinucleate and a proportion contained pale cytoplasmic vacuoles which, in a small minority of cells were very prominent. The synovial surface was, in

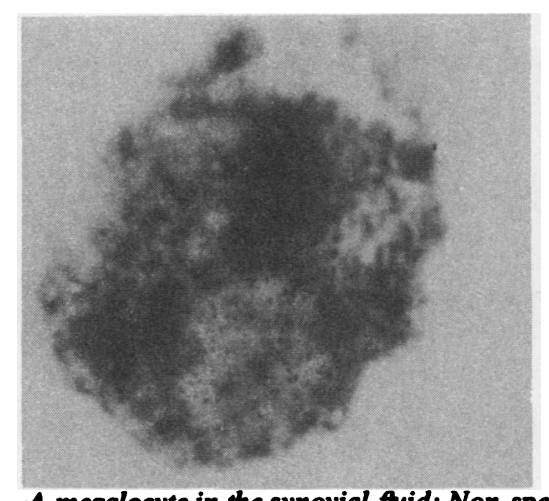

Fig. 1 A megalocyte in the synovial fuid: Non-specific esterase $\times 700$. 


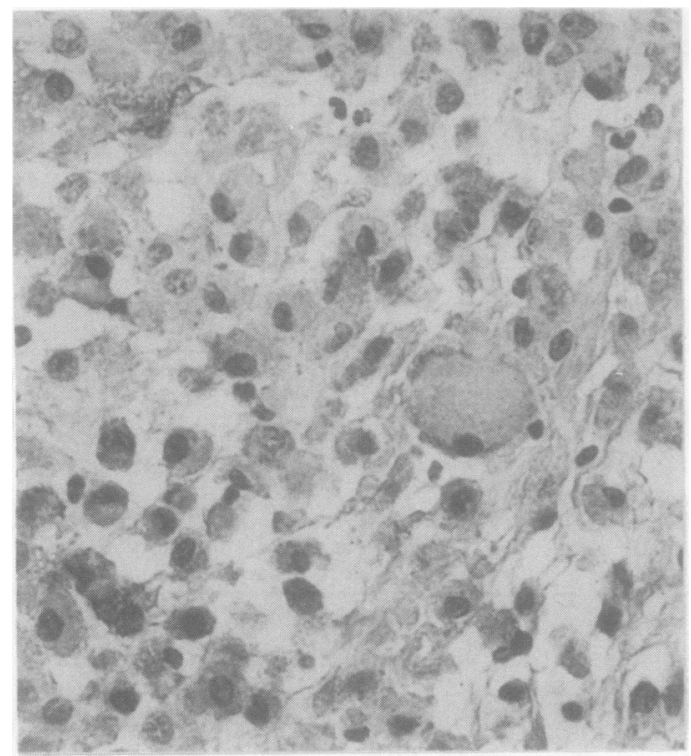

Fig. 2 The megalocytic and histiocytic in fitrate in the synovium. Haematoxylin and eosin $\times 200$.

places, covered with fibrinous deposits and deep to the intima a scanty lymphocyte, plasma cell and histiocyte infiltrate could be seen.

Most of the megalocytes contained PAS-positive granules and globules which were diastase-resistant; these cells also stained strongly for muramidase (Fig. 3) and moderately with alpha-1-antitrypsin. The pattern of immunocytochemical staining varied

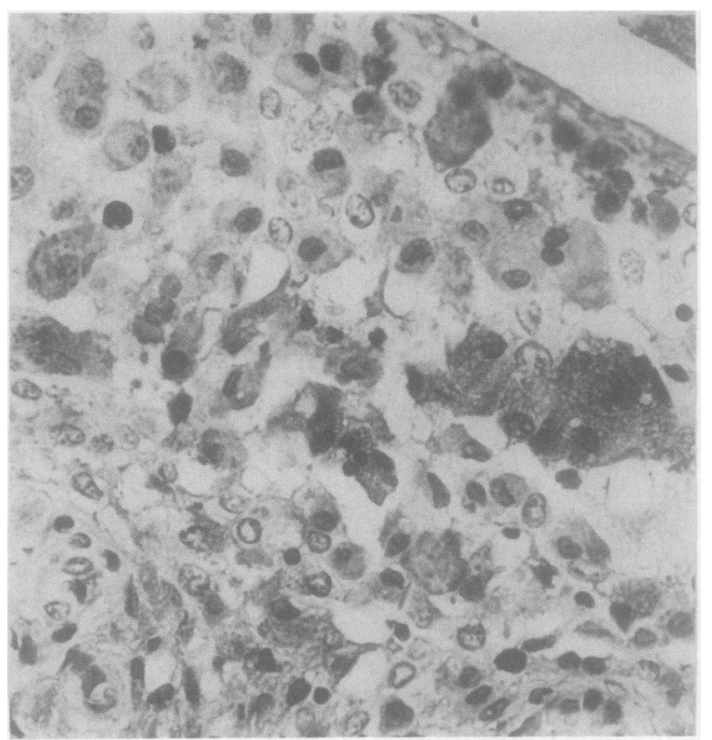

Fig. 3 Muramidase positive cells in the synovium. Muramidase $\times 200$.

from cell to cell; in most the activity lay in a reticular pattern throughout the cytoplasm, others stained uniformly and a third group of cells exhibited a dense rim of stain beneath the surface membrane surrounding a large central vacuole.

\section{Electron microscopy}

In areas the synovium appeared normal but else-

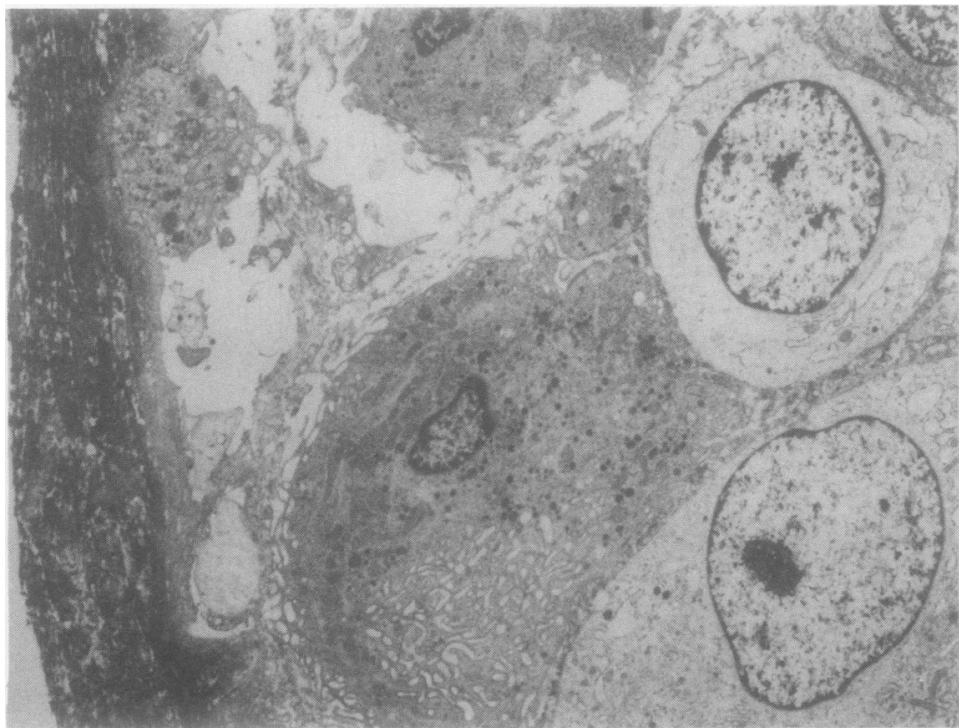

Fig. 4 A low power electron-micrograph showing surface' fibrin, pale and dark cells $\times 1100$. 


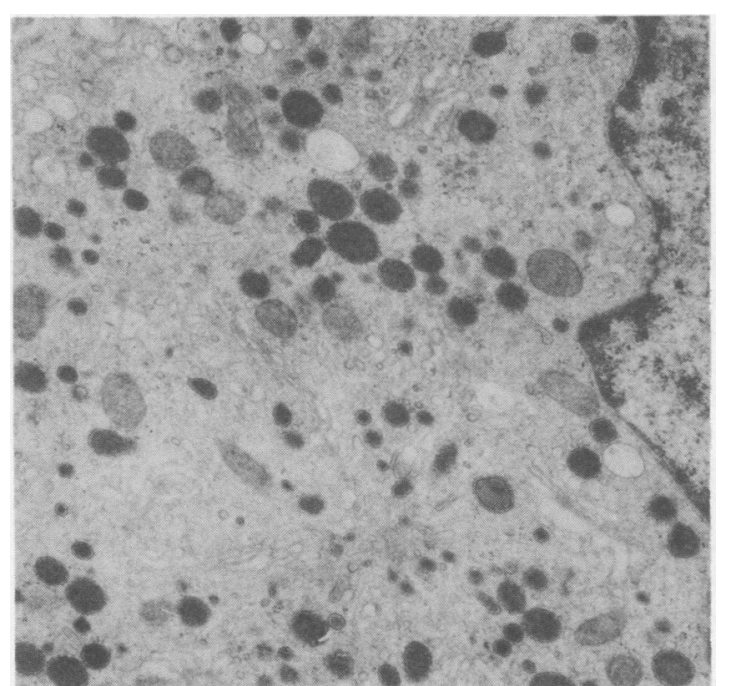

Fig. 5 The cytoplasm of a megalocyte with osmiophilic granules and a large stellate Golgi apparatus $\times 11000$.

where the surface was covered in fibrin, deep to which could be seen aggregations of cells of which there appeared to be two types, pale and dark (Fig. 4 ), the dark outnumbering the pale by a factor of twenty.
The pale cells had large ovoid nuclei with finely dispersed chromatin and prominent nucleoli. The electron-lucent cytoplasm contained many cisternae of rough endoplasmic reticulum, some of which were dilated by slightly flocculent contents. Mitochondria were numerous with a somewhat electron dense matrix and lighter cristae. Golgi bodies and associated vacuoles were also present together with occasional inclusion bodies which were generally smooth, round and electron-dense, and some fat droplets. Skeins of fine filaments were interspersed between organelles. These cells tended to have a smooth outline and sometimes formed nests of closely fitting groups of cells. Collagen fibres and flocculent material were often found in close proximity to the cell membranes.

In contrast, the dark cells exhibited considerable variation in size, over $90 \%$ being very large. This latter group corresponded with the megalocytes described light microscopically in number, position and dimensions. Despite the variability in size the ultrastructure of the cells was relatively uniform. The nuclei were irregular, containing condensed chromatin and frequently central nucleoli. A striking characteristic of this cell type was the abundance of electron-dense droplets which were scattered throughout the cytoplasm. Small vacuoles of

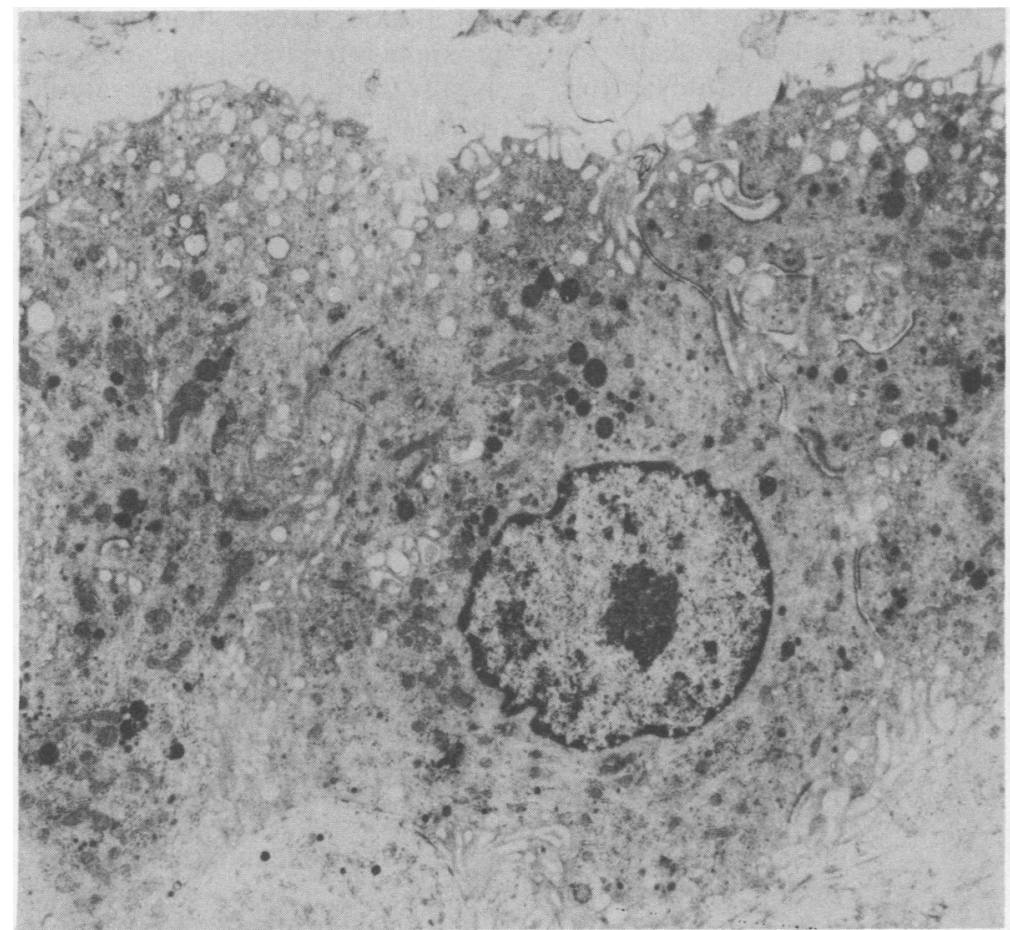

Fig. 6 Three megalocytes linked by desmosomes and showing areas where the distinction between the cytoplasm of adjacent cells is lost $\times 3500$. 
medium electron density appeared to originate from the numerous Golgi bodies which often formed a stellate pattern with groups of cisternae and vacuoles radiating from a central point (Fig. 5).

Droplet electron density appeared to increase with size and the contents of some were heterogeneous. Smooth and coated vesicles were also associated with the Golgi vacuoles and mitochondria were numerous. Rough endoplasmic reticulum was sparse in many of these cells, whilst smooth surfaced vacuoles were often present in remarkable configurations, particularly near the plasma membrane, where they merged with complex infoldings of the surface to produce a lace-like effect. Many of these electron-dense cells appeared to be in the process of fusion and possessed local densities on opposing plasma membranes, sometimes exhibiting a fine electron dense line running between them, and on others disappearing completely to reveal apparent cytoplasmic continuity between adjoining cells (Fig. 6 ). The multinucleate cells generally displayed a similar cytoplasmic ultrastructure to the dark cells except that they often contained large pale vacuoles and their surface was smoother.

Cells intermediate in structure between pale and dark cells with well developed endoplasmic reticulum as well as numerous secretory droplets were also occasionally found.

Ultrastructurally, the dark cells closely resemble the type A synoviocytes described by Ghadially ${ }^{9}$ and the pale cells are similar to his type B synoviocytes.

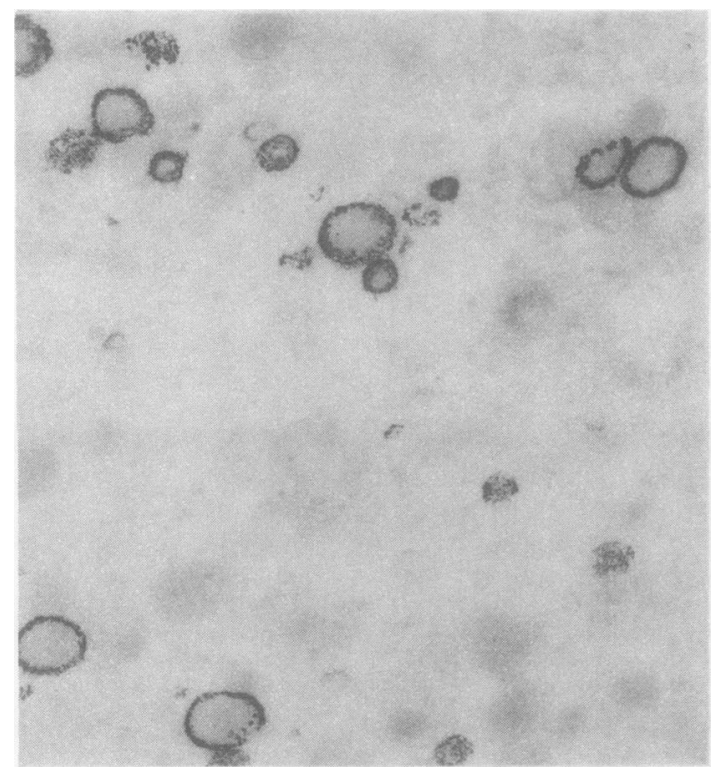

Fig. 7 Aryl sulphatase positive rim to the osmiophilic granules of the megalocytes $\times 19000$.
The ultrahistochemical studies showed the presence of both acid phosphatase and aryl sulphatase activity within the tissue. Both reaction products were restricted to the osmiophilic granules of the dark and intermediate cells and each had a distinctive distribution within the granule. Acid phosphatase activity was found within the centre of the granule and an unreactive halo was present immediately beneath the limiting membrane, while the region of the granule occupied by this halo showed aryl sulphatase activity, the centre being unreactive (Fig. 7).

\section{Discussion}

Multicentric reticulohistiocytosis was first described in 1937 as a non-diabetic cutaneous xanthomatosis. ${ }^{10}$ It has subsequently received numerous synonyms, ${ }^{11}$ including lipoid rheumatism, normocholesterolaemic xanthomatosis, reticulohistiocytic granuloma, reticulohistiocytoma, giant cell reticulohistiocytosis, reticulohistiocytosis and multicentric reticulohistiocytosis. As little is known of the pathogenesis of this disease, the last, purely descriptive term is probably the most apt.

The clinical picture is frequently characteristic, ${ }^{3}$ the patient presenting with the insidious onset of a polyarthropathy, usually of rheumatoid type and distribution, except that the distal as well as the proximal interphalangeal joints are involved. This is usually followed, at an interval ranging from days to years, by a nodular maculopapular mucocutaneous eruption. The disease occurs more commonly in women that men, and the mean age of onset is in the fifth decade.

Although rare, the disease is clinically significant because of two associations. Firstly, the arthropathy usually evolves into a severe, deforming, erosive arthritis which is unresponsive to treatment, ${ }^{12}$ and secondly, there is an associated underlying malignancy in $25 \%$ of cases. ${ }^{1}$

Although multicentric reticulohistiocytosis may often be suspected clinically, histopathological confirmation is usually required. Histologically the skin, mucous membrane and synovial lesions are said to be very similar, the main feature being the infiltration of the tissue by giant histiocyte-like cells up to $100 \mu \mathrm{m}$ in diameter with either abnormally large or multiple nuclei. These cells were initially described as having a characteristic ground glass appearance, ${ }^{13}$ although as the number of published cases increases, it is becoming apparent that an absence of this feature in no way precludes the diagnosis.

Histochemical studies have shown the cytoplasm always to contain diastase-resistant PAS-positive 
material ${ }^{3}$ and frequently lipids which stain variously with Sudan Black B, Sudan III and IV and Oil Red 0.12 The presence of fat within the megalocytic cells has lead to speculation that the disease may represent a lipidosis but this view is being increasingly challenged and evidence is accumulating that it is a coincidental finding probably related to cell degeneration.

Recognisable, relatively small histiocytes are also found in the lesions as are lymphocytes, often clustered about vessels with distinctive endothelial cells as described in other sites of lymphocyte migration into tissue. ${ }^{14}$

The histological appearances of the synovial lesions from our patient are in keeping with descriptions of both the skin and synovium in multicentric reticulohistiocytosis and we have been able to demonstrate immunocytochemically the histiocytic origin of the large cells.

Our electron microscopic findings also coincide with the descriptions of others. ${ }^{15}$ The most noticeable feature of the megalocytes is the large number of osmiophilic membrane-bound granules in the cytoplasm, often closely associated with a single large stellate Golgi apparatus. Tani and his coworkers ${ }^{16}$ have demonstrated in the skin that these granules are both the site of PAS positivity and are rich in acid phosphatase activity. They have demonstrated this enzyme to be concentrated in the centre of each granule, leaving an unstained "halo" immediately beneath the unit membrane, an appearance described by others as being distinctive for lysosomes. ${ }^{17}$ We have demonstrated this unstained region to contain aryl sulphatase, another enzyme associated with lysosomes. This finding may well indicate that the enzyme is membrane bound at this site.

From theirobservations, Tani et al have proposed that the lesions of multicentric reticulohistiocytosis are very similar to experimental granulomata, with small activated histiocytes undergoing a morphological and functional transition to autophagocytic multinucleate giant cells. They maintain that the small population of relatively normal looking histiocytes represents the activated heterophagic cells which then gradually transform into intermediate cells which fuse and undergo degeneration, finally becoming autophagocytic fat-laden giant cells.

In the synovium we have noted cells apparently fusing to form multinucleate giant cells and the sequence of events could well be similar at this site. That the fusing cells are ultrastructurally similar to type A synoviocytes, thought to be the local synovial macrophages, ${ }^{9}$ may also support the view that multicentric reticulohistiocytosis is a bizarre granulomatous response, presumably to an, as yet unidentified, environmental stimulus. This possibility has been pursued before ${ }^{3}$ and although an unexpectedly large number of patients displayed tuberculin skin sensitivity, no acid fast bacilli could be demonstrated in the lesions.

An active histiocytic inflammatory process would explain many of the synovial fluid findings which initially appear contradictory. The most unusual feature of the synovial fluid analysis is the combination of low cell count (usually associated with a noninflammatory arthropathy) and poor mucin clot formation (which is generally seen in active intraarticular inflammation). The explanation of this apparent anomaly may be that the release of proteolytic enzymes from small activated histiocytes and degenerating megalocytes is responsible for the inflammatory changes seen in the joint, including fibrin deposition on the synovial surface, interference with hyaluronic acid production or polymerisation resulting in poor mucin clot formation, and erosion of cartilage and bone leading to the severe destructive arthropathy, a mechanism very similar to that postulated for some of the erosive crystal arthropathies.

The size and nature of most of the infiltrating cells would prevent them from entering the synovial fluid, thus explaining the low cell count, although some histiocytes, rare megalocytes and occasional lymphocytes, might be expected to be present in the fluid, as indeed they are. That the large cells in the fluid are probably derived from the synovial megalocytes is suggested not only by their distinctive appearance, with copious cytoplasm and large nuclei, but also by their cytochemistry with the fluid cells displaying the dense granular cytoplasmic reaction product for non-specific esterase characteristic of cells of the monocyte/macrophage series, and the synovial cells the muramidase and alpha-1antitrypsin immunocytochemistry also characteristic of this cell line.

This is only the second report in the English literature in which the synovial fluid from a patient with multicentric reticulohistiocytosis has been subjected to a differential cell count. In the previous account, ${ }^{11}$ the findings were very similar to our own. A review of the last 500 synovial fluids processed in this laboratory failed to reveal any other exhibiting a low white cell count with predominantly lymphocyte and monocyte cell populations associated with poor mucin clot formation, and bizarre large macrophages.

These findings are so characteristic that it may be possible to use synovial fluid aspiration and analysis, a relatively simple and non-invasive technique, as a method of diagnosing this rare but important disease early in its progression even in those patients in 
whom the onset of arthritis antedates the rest of the clinical manifestations.

We wish to thank Professor $\mathbf{J}$ Ball for his help and advice in preparing this paper. Dr Jones is supported by the Hadwen Trust for Human Research to whom we are very grateful.

\section{References}

' Catteral MD. Multicentric reticulohistiocytosis-a review of eight cases. Clin Exp Dermatol 1980;5:267-79.

2 Dixon A St J. "Rheumatoid arthritis" with negative serological reaction. Ann Rheum Dis 1960;19:209-28.

${ }^{3}$ Barrow MV, Holubar K. Multicentric reticulohistiocytosis-a review of 33 patients. Medicine 1960;48:287-305.

4 Yam LT, Li CV, Crosby WH. Cytochemical identification of monocytes and granulocytes. Am J Clin Pathol 1971;55:28390.

${ }^{5}$ Freemont AJ, Davies JSD. Acid esterase activity in lymphocytes and other cells: a comparison of six-naphthyl based substrates. Med Lab Sci 1982;39:05-7.

- Graham RC, Karnovsky MJ. The early stages of absorption of injected horseradish peroxidase in the proximal tubules of mouse kidney: ultrastructural cytochemistry by a new technique. J Histochem Cytochem 1966;14:291-6.

' Jones CJP, Fox H. An ultrastructural and ultrahistochemical study of the placenta of the diabetic woman. J Pathol 1976;119:91-9.
- Jones CJP, Fox H. An ultrahistochemical study of the distribution of acid and alkaline phosphatase in placentae from normal and complicated pregnancies. J Pathol 1976;118:143-51.

- Ghadially FN, Roy S. Ultrastructure of synovial joints in health and disease. London: Butterworths, 1969.

10 Weber FP, Freudenthal W. Nodular non-diabetic cutaneous xanthomatosis with hypercholestrolaemia and atypical histological features. Proc R Soc Med 1937;30:522-6.

"Rooney PJ, Ballantyre D, Watson Buchanon W. Clinics in rheumatological diseases. 1:1 $\rightarrow$ heumatological manifestations of systemic diseases. London: WB Saunders, 1975.

${ }^{12}$ Krey PR, Comerford FR, Cohen AS. Multicentric reticulohistiocytosis. Fine structural analysis of the synovium and synovial fluid cells. Arthritis Rheum 1974;17:615-33.

${ }^{13}$ Davies BT, Wood SR. So-called reticulohistiocytoma of the skin. Comparison of two distinct types. British Journal of Dermatology and Syphilology 1955;67:205-11.

${ }^{14}$ Freemont AJ. A possible route for lymphocyte migration into diseased tissue. J Clin Pathol 1983;36:161-6.

is Albert J, Bruce W, Allen AC, Blank H. Lipoid dermatoarthritis. Reticulohistiocytoma of the skin and joints. Am J Med 1960;28:661-7.

${ }^{16}$ Tani M, Hari K, Nakanishi T, Iwasaki T, Ogawa Y, Jimbo T. Multicentric reticulohistiocytosis. Electron microscopic and ultracytochemical studies. Arch Dermatol 1981;117:495-9.

17 Daerns WT, Wisse E, Brederoo P. Electron microscopy of the vascular apparatus. Lysosomes in Biology and Pathology. Vol 1. Amsterdam: North-Holland Publishing Co, 1969.

Requests for reprints to: Dr AJ Freemont, Department of Pathology, University of Manchester, Stopford Building, Oxford Road, Manchester M13 9PT, England. 